\title{
Identificación de Bacterias que afectan el establecimiento in vitro de segmentos nodales de Guadua angustifolia Kunth
}

\section{Identification of bacteria affecting in vitro establishment of nodal segments of Guadua angustifolia Kunth}

\author{
Lorena Alexandra Ramírez*, Sandra Milena Castaño* Rodolfo López ${ }^{* *}$
}

Recibido: Septiembre 30 de 2009

Aceptado: Diciembre 1 de 2009

Correspondencia: Laboratorio de Biotecnología Vegetal, Universidad del Quindío avenida Bolivar Calle 12 norte Armenia Quindío. Email: rlopez@uniquindio.edu.co

\section{RESUMEN.}

El cultivo in vitro de segmentos nodales (medio y basal), de ramas de chusquines de Guadua angustifolia Kunth., presenta un alto grado de contaminación por bacterias afectando su micropropagación, en la fase de establecimiento. Para la identificación de las bacterias contaminantes, se seleccionaron siete grupos con características de aspecto y color, a las que se les aplicaron medios de cultivo diferenciales, como los utilizados para géneros bacterianos fitopatógenos, como: YDC; B de King; D1; y D5; además, se efectuaron pruebas bioquímicas para confirmar la identificación, como: tinción de Gram; prueba de Hugh y Leifson; Indol; y motilidad. Mediante la presente investigación, se llegó a la identificación de los géneros: Xanthomonas, Pseudomonas, Agrobacterium, y una asociación entre Erwinia-Pseudomonas. El género Pseudomonas fue el principal agente contaminante para los segmentos nodales basales (26.8\%) y la asociación Erwinia-Pseudomonas para segmentos nodales medios (26.6\%). La sensibilidad a los antibióticos por parte de las bacterias, se determinó a través del antibiograma, donde los géneros encontrados presentaron sensibilidad a Amikacina, Gentamicina y Vancomicina.

Palabras clave: in vitro, segmentos nodales, Guadua angustifolia, fitopatógenos, antibióticos.

\section{ABSTRACT}

The in vitro culture of nodal segments (medium and basal) of branches chusquines Guadua angustifolia Kunth, has a high degree of bacterial contamination, affecting their micropropagation, in the establishment phase. For identification of the contaminating bacteria, seven groups with aroma and color characteristics were selected, which were applied differential culture media, such as those used for phytopathogenic bacterial genera, such as: YDC, King B, D1 and D5. In addition, biochemical tests were performed to confirm identification such as: gram stain, Hugh and Leifson test, Indol; and motility. This investigation led to the identification of the genera: Xanthomonas, Pseudomonas, Agrobacterium, and Erwinia-Pseudomonas partnership. The genus Pseudomonas was the main pollutant for basal nodal segments (26.8\%) and Erwinia-Pseudomonas association for media nodal segments (26.6\%). The sensitivity to antibiotics by bacteria, were determined by antibiogram, where genera was found to be sensible to Amikacin, Gentamicin and Vancomycin.

Keywords: in vitro, nodal segments, Guadua angustifolia, plant pathogens, antibiotics.

\section{INTRODUCCIÓN}

as técnicas de cultivo in vitro han desarrollado una exitosa y rápida propagación asexual de un gran número de especies vegetales, donde uno de los requisitos básicos es mantener los cultivos libres de microorganismos contaminantes $(1,2)$.
El efecto de los microorganismos contaminantes sobre las plantas in vitro es considerable, si se tiene en cuenta que compiten con ellas por los nutrientes del medio de cultivo y proporcionan daños directos e indirectos por la colonización de sus tejidos y la expulsión al medio de metabolitos tóxicos (2-4).

\footnotetext{
* Programa Licenciatura en Biología y Educación Ambiental. Facultad de Educación. Universidad del Quindío

**Laboratorio de Biotecnología Vegetal-CIBUQ. Universidad del Quindío.
} 
Los microorganismos ambientales (no patógenos), no representan un perjuicio significativo para la planta, pero en condiciones in vitro, óptimas en nutrientes, se desarrollan hasta extremos que resultan perjudiciales ocasionando la pérdida total del material vegetal, por la colonización de los tejidos y la expulsión al medio de metabolitos tóxicos (5).

Por ser la guadua (Guadua angustifolia Kunth) una especie de amplia distribución en América, además de sus propiedades físicas y mecánicas, y por cumplir en Colombia un importante papel ambiental, cultural y económico $(6,7)$, se han explorado numerosos métodos de propagación masiva, incluidos los procesos de micropropagación (8-10).

Sin embargo, en la fase de establecimiento de la micropropagación de guadua, varios autores reportan altos porcentajes de contaminación provocados por microorganismos, con énfasis en los deterioros causados en el material vegetal por las bacterias (9-14).

Pero en los trabajos de micropropagación de G. angustifolia, sólo se reporta la identificación de la bacteria Bacillus sp. como causante de la contaminación en la fase de establecimiento in vitro (15).

Por tal razón, el presente trabajo de investigación se propuso identificar las bacterias contaminantes que afectan la micropropagación de los segmentos nodales de $G$. angustifolia, para advertir sobre la necesidad de medidas de control y prevención, en la fase de establecimiento del cultivo in vitro de esta especie forestal.

\section{MATERIALES Y MÉTODOS.}

\section{Fase de establecimiento in vitro}

El estudio se realizó en el Laboratorio de Biotecnología de la Corporación Regional del Quindío, del Centro Nacional para el Estudio del Bambú-guadua, ubicado en el municipio de Córdoba-Quindío; y en el Centro de Investigaciones Biomédicas "Manuel Elkin Patarroyo" de la Universidad del Quindío, de la ciudad de Armenia-Quindío.

En el Laboratorio de Biotecnología se realizó la siembra de explantes de G. angustifolia, los cuales fueron obtenidos de ramas de chusquínes de guadua que permanecieron bajo condiciones de invernadero; de tales ramas se seleccionaron como: segmento nodal basal y segmento nodal medio; explantes que se han recomendado como los más promisorios en la micropropagación de esta especie vegetal (9).

Los explantes fueron sometidos a un lavado con solución de detergente comercial por 15 min; luego en cabina de flujo laminar se sumergieron en alcohol del $70 \%$ durante $1 \mathrm{~min}$; posteriormente se sometieron a exposición de una solución de $\mathrm{NaClO}$ al $3 \%$ por $15 \mathrm{~min}$; seguidamente se realizaron tres enjuagues con agua destilada estéril; luego fueron inoculados en medio de cultivo (16) suplementado con la citoquinina BAP, a razón de $3.0 \mathrm{mg} \mathrm{L}-1$. Los explantes inoculados se llevaron a cámara de crecimiento, donde permanecieron por 30 días a condiciones de fotoperíodo por 12 horas (13).

Se realizó la siembra de 2000 segmentos nodales basales y 2000 segmentos nodales medios; a las dos semanas de cultivo, de los materiales libres de contaminación por hongos, se tomaron al azar 619 segmentos nodales basales $y$ 619 segmentos nodales medios. Dichos explantes se tuvieron en observación por un período de cuatro semanas; para un mejor análisis, los explantes se organizaron por grupos según la sintomatología exhibida, tanto por el explante como por la apariencia y desarrollo de la bacteria en el medio de cultivo; de cada grupo constituido, se seleccionaron los materiales para la fase del estudio microbiológico. Para estimar el tiempo promedio de aparición y el porcentaje de contaminación por acción de las bacterias, sobre los dos tipos de segmentos nodales de $\mathrm{G}$. angustifolia, se realizó el análisis estadístico mediante el programa Statistix 7.0.

\section{Fase de estudio microbiológico.}

Las pruebas de identificación de bacterias se llevaron a cabo en el Centro de Investigaciones Biomédicas "Manuel Elkín Patarroyo" de la Universidad del Quindío. Las bacterias de los grupos de explantes que presentaron contaminación, se dispusieron en caldo peptonado a $28{ }^{\circ} \mathrm{C}$ por $30 \mathrm{~min}$. Seguidamente se realizó la purificación de las colonias bacterianas (17), que fueron sembradas por agotamiento en agar tripticasa de soya a $28^{\circ} \mathrm{C}$ por 24 horas, hasta obtener un cultivo puro (18); las colonias de bacterias aisladas se sembraron en medio de cultivo, según el esquema de Shaad (19), con aumento del medio D5 (20) y la prueba de Hugh y Leifson (21).

Se les realizó tinción de Gram, donde las bacterias gram negativas, fueron sembradas en el medio YDC; las colonias que se tornaron de color amarillo, fueron expuestas a la prueba de Hugh y Leifson (21), que indicó su tipo de respiración (oxidativa o fermentativa). Cuando las colonias presentaron color crema, se sembraron en medio B de King (22), las cuales presentaron (o no) fluorescencia bajo la observación de luz ultravioleta. Posteriormente se les realizó la prueba de Hugh y Leifson (21), para identificar su respiración (oxidativa o fermentativa); con el fin de 
diferenciar los géneros, se sembraron en medio de cultivo D1 para observar su crecimiento; además, se llevaron a cabo las pruebas bioquímicas que permitieran la confirmación de los géneros.

El antibiograma se realizó mediante la prueba de Difusión de disco, o Prueba de Kirby-Bauer $(18,23,24)$, para determinar la sensibilidad de las bacterias aisladas e identificadas, a la presencia de diferentes antimicrobianos (tabla 1).

Tabla 1. Antibiogramas utilizados para la identificación de los géneros de bacterias.

\begin{tabular}{|l|c|}
\hline \multicolumn{1}{|c|}{ SENSIDISCOS } & CANT IDAD \\
\hline Penicilina & $10 \mathrm{mcg}$ \\
\hline $\begin{array}{l}\text { Amoxicilina - ac. } \\
\text { clavulónico }\end{array}$ & $20 / 10 \mathrm{mcg}$ \\
\hline Cefalotina & $30 \mathrm{mcg}$ \\
\hline Cefotaxima & $30 \mathrm{mcg}$ \\
\hline Ceftriaxona & $30 \mathrm{mcg}$ \\
\hline Vancomicina & $25 \mathrm{mcg}$ \\
\hline Amoxicilina & $10 / 10 \mathrm{mcg}$ \\
\hline Ampicilina - sulbactam & $15 \mathrm{mcg}$ \\
\hline Eritromicina & $5 \mathrm{mcg}$ \\
\hline Ciprofloxacino & $10 \mathrm{mcg}$ \\
\hline Gentamicina & $30 \mathrm{mcg}$ \\
\hline Amikacina & $10 \mathrm{mcg}$ \\
\hline Ampicilina & $10 \mathrm{mcg}$ \\
\hline Norfloxacino & \\
\hline
\end{tabular}

\section{RESULTADOS Y DISCUSIÓN.}

Los segmentos nodales medio y basal cultivados in vitro, libres de contaminación por hongos, fueron evaluados durante cuatro semanas para observar el efecto de la contaminación por bacterias; los segmentos medios mostraron una contaminación del 56.5\%, mientras que los basales indicaron una contaminación bacterial del 52\% (tabla 2). De acuerdo a la sintomatología exhibida, se organizaron siete grupos para escoger dos muestras por grupo, con las que se procedió a realizar su identificación. Las condiciones de $\mathrm{pH}$ y humedad de los cultivos in vitro, permitieron el desarrollo de estos microorganismos, ya que las bacterias toleran niveles de $\mathrm{pH}$ neutros $\mathrm{y}$ requieren de humedad relativa alta (25).
Tabla 2. Contaminación de segmentos nodales de G. angustifolia en condiciones in vitro.

\begin{tabular}{|c|c|c|c|}
\hline $\begin{array}{c}\text { TIPO DE } \\
\text { EXPLANTE }\end{array}$ & $\begin{array}{c}\text { SIEMBRA } \\
\text { TOTAL }\end{array}$ & $\begin{array}{c}\text { CANTIDAD } \\
\text { CONTAMINADOS }\end{array}$ & $\begin{array}{c}\% \\
\text { CONTAMINACION }\end{array}$ \\
\hline $\begin{array}{c}\text { Segmento } \\
\text { MEDIO }\end{array}$ & 619 & 359 & 56.5 \\
\hline $\begin{array}{c}\text { Segmento } \\
\text { BASAL }\end{array}$ & 619 & 322 & 52 \\
\hline TOTAL & 1238 & 681 & 54 \\
\hline
\end{tabular}

Aislamiento e Identificación.

A partir de la lectura de los medios diferenciales para la identificación de los géneros bacterianos, se encontró la presencia de Xanthomonas, Erwinia, Pseudomonas y Agrobacterium, tal como se describe en la tabla 3. Estos resultados coincidieron con las características de crecimiento de las colonias reportadas por varios autores $(20,26)$, pero difieren de lo descrito por Cruz et al. (15), quienes identificaron sólo al género Bacillus sp. Por tanto, este trabajo se convierte en el primer reporte de identificación de las bacterias mencionadas, como contaminantes de la fase de establecimiento in vitro de $G$. angustifolia.

Las colonias de bacterias que se identificaron como Gram negativas, por el método de tinción de Gram, fueron cultivadas en medio YDC; aquellas cuyo crecimiento presentó color amarillo fueron sometidas a la prueba de Hugh y Leifson (21), donde el género Xanthomonas exhibió su respiración característica de tipo oxidativa (tabla 3).

Tal condición se confirmó cuando este tipo de bacterias presentó crecimiento en medio D5 (20), determinando así el género Xanthomonas. El aspecto de Xanthomonas en cultivo in vitro de G. angustifolia, se manifestó de dos maneras: 1 ) presentó crecimiento alrededor del explante y colonizó todo el medio de cultivo, acompañado de un color amarillo mostaza; 2) creció a partir del explante formando camino y rodeando el borde del frasco, con coloración blanco hialino, haciéndose visible por la base del frasco de siembra.

Igualmente, aquellas colonias que se tornaron de color amarillo y que mediante la aplicación de la prueba de Hugh y Leifson (21), dieron como respuesta la respiración fermentativa, correspondieron a la característica típica del género Erwinia (tabla 3).

Además, se identificó una asociación entre ErwiniaPseudomonas, que en cultivo in vitro se caracterizó por presentar crecimiento alrededor del segmento nodal, 
Tabla 3. Resultados de pruebas de identificación para cada una de las bacterias.

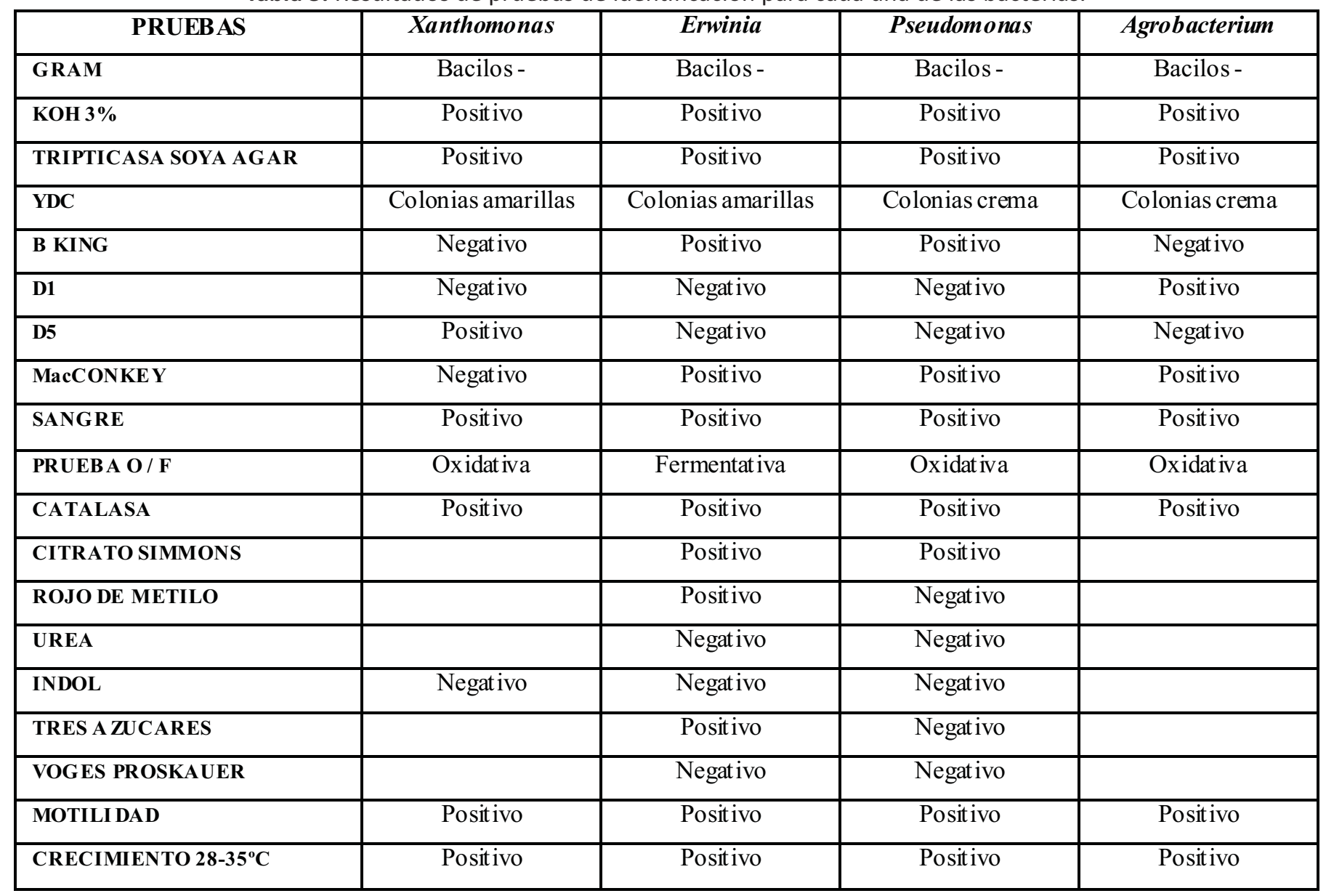

acompañado de una estela en la parte basal del explante, de color crema claro, que después de varios días tornó al medio de color amarillo- verdoso tenue. A las demás colonias que se tornaron de color amarillo, y fueron sometidas a la prueba de Hugh y Leifson (21), dando como respuesta la respiración oxidativa, correspondieron a los géneros Pseudomonas (tabla 3) y Agrobacterium (tabla 3); para diferenciar estos dos géneros, se sembraron en el medio de cultivo D1, donde Pseudomonas no exhibió crecimiento.

La presencia de Pseudomonas sobre los explantes de G. angustifolia, en condiciones in vitro, manifestó varios aspectos: 1) se caracterizó por poner turbio el medio de cultivo, de color crema oscuro, creciendo a partir del explante; 2) presentó la misma característica anterior pero acompañado de una elevación en burbujas (por producción de gas); 3) al principio de la contaminación, tuvo características iguales a la primera manifestación, pero después de dos días pigmentó el medio de cultivo de color azul-verdoso. Todas colonizaron por completo el medio de cultivo y no hubo necesidad de observarlas a la luz.

Los géneros identificados concuerdan con lo reportado por
Leifert et al. (5), quienes aislaron Erwinia y Pseudomonas, como patógenos de diferentes especies de vegetales. Además, Pseudomonas se presentó en mayor porcentaje de contaminación en segmentos basales, lo que concuerda con lo reportado en otros trabajos, donde se ha indicado que este microorganismo es el más frecuente en la naturaleza, encontrándose principalmente en el agua, la tierra y las plantas, lo que indica que la contaminación proviene del suelo (27), lugar donde están sembradas las plantas madre (chusquines de guadua).

Cuando las colonias se cultivaron en medio D1, Agrobacterium fue capaz de presentar crecimiento, diferenciándose de Pseudomonas. En el cultivo in vitro de segmentos nodales de G. angustifolia, Agrobacterium manifestó crecimiento alrededor del explante, el cual se observó desde el fondo del envase hacia la luz, de color blanco hialino y con el transcurso de varios días se tornó de un color rosado claro. Del total de explantes (tabla 4), el género Pseudomonas contaminó un total de 141 segmentos medios y 165 basales; en los segmentos medios, la asociación de los géneros Erwinia-Pseudomonas contaminó 164 explantes mientras que en los basales la contaminación 
fue de 125 explantes; aunque su presencia se manifestó de manera conjunta, ellas fueron aisladas e identificadas como géneros diferentes en la fase de laboratorio microbiológic; Xanthomonas contaminó 51 segmentos medios y 27 basales.

Tabla 4. Contaminación por bacterias que afectaron el cultivo in vitro de segmentos nodales (medio y basal) de G. angustifolia.

\begin{tabular}{|c|c|c|c|c|}
\hline \multirow{2}{*}{ Géneros } & \multicolumn{2}{|c|}{$\begin{array}{c}\text { Totales Segmento } \\
\text { Contaminado }\end{array}$} & Porcentaje & Porcentaje \\
\hline \multirow{2}{*}{ Pseudomonas } & Medio & Basal & Medio & Basal \\
\cline { 2 - 5 } & 141 & 165 & 22.8 & 26.8 \\
\hline Xanthomonas & 51 & 27 & 8.2 & 4.4 \\
\hline $\begin{array}{c}\text { Pseudomonas- } \\
\text { Eminia }\end{array}$ & 164 & 125 & 26.6 & 20.2 \\
\hline Agrobacterium & 3 & 5 & 0.3 & 0.6 \\
\hline Total & 359 & 322 & 57.9 & 52.0 \\
\hline
\end{tabular}

Agrobacterium contaminó 3 explantes medios y 5 basales, por lo que se consideró poco relevante su participación en los procesos de contaminación de segmentos nodales de guadua, en la fase de establecimiento in vitro.

Así, el segmento nodal medio presentó mayor contaminación por causa de la asociación Erwinia-Pseudomonas, mientras que el segmento basal presentó mayor contaminación a causa de Pseudomonas; varios autores han propuesto que estos organismos son los más frecuentes tanto en el ambiente como en la planta $(19,28-30)$.

\section{Tiempo de contaminación}

Para el segmento nodal medio (tabla 5), el tiempo promedio de contaminación de los cultivos in vitro, fue: 7.1 días para Pseudomonas, 7.5 días para Xanthomonas y 6.8 días para Pseudomonas - Erwinia. Según el intervalo del $95 \%$ de confianza, no existen diferencias significativas en por el tiempo de aparición de la contaminación entre los tres géneros; es decir, cada género de bacterias contamina los cultivos de guadua en condiciones in vitro aproximadamente al mismo tiempo.

El género Agrobacterium fue poco frecuente $y$ de contaminación retardada en el cultivo in vitro de segmentos nodales de guadua, con una aparición entre 9 y 11 días; considerándose este género poco significativo al momento del análisis estadístico.
En cuanto al segmento nodal basal, el tiempo promedio de aparición de la contaminación de los cultivos in vitro, fue: 5.4 días para Pseudomonas, 6.4 días para Xanthomonas y 7.4 días para la asociación de Pseudomonas - Erwinia. Además, según el intervalo del $95 \%$ de confianza, hay diferencias en el tiempo medio de aparición, entre los tres géneros; es decir, la asociación Pseudomonas - Erwinia muestra diferencias significativas con respecto a Pseudomonas y Xanthomonas.

Tabla 5. Tiempo de contaminación por bacterias que afectan el cultivo in vitro del segmento nodal medio de $G$. angustifolia.

\begin{tabular}{|l|c|c|c|c|c|c|}
\hline \multicolumn{1}{|c|}{ Géneros } & $\mathbf{n}$ & $\bar{x}$ & $\mathbf{S}$ & $\begin{array}{c}\text { Intervalo } \\
\text { del } \\
\mathbf{9 5 \%}\end{array}$ & $\begin{array}{c}\text { Tiemp } \\
\mathbf{o} \\
\text { mínim } \\
\text { o d́́s }\end{array}$ & $\begin{array}{c}\text { Tiempo } \\
\text { máxim } \\
\text { o dís }\end{array}$ \\
\hline Peudomonas & 141 & 7.1 & 4.5 & $6.3-7.8$ & 2 & 21 \\
\hline Xanthomonas & 51 & 7.5 & 4.2 & $6.3-8.6$ & 3 & 21 \\
\hline $\begin{array}{l}\text { Pseudomonas- } \\
\text { Erwinia }\end{array}$ & 165 & 6.8 & 3.9 & $6.2-7.3$ & 3 & 21 \\
\hline Agrobacterium & 2 & & & & 9 & 11 \\
\hline
\end{tabular}

El género Agrobacterium fue poco frecuente y de contaminación retardada en el cultivo in vitro de yemas basales de guadua, con una aparición entre 7 y 21 días, por lo que se consideró este género poco significativo en el análisis estadístico.

Tabla 6. Tiempo de contaminación por bacterias que afectan el cultivo in vitro del segmento nodal basal de $G$. angustifolia.

\begin{tabular}{|l|c|c|c|c|c|c|}
\hline \multicolumn{1}{|c|}{ Géneros } & $\mathbf{n}$ & $\bar{x}$ & $\mathbf{S}$ & $\begin{array}{c}\text { Intervalo } \\
\text { del } \\
\mathbf{9 5 \%}\end{array}$ & $\begin{array}{c}\text { Tiempo } \\
\text { mínimo } \\
\text { dís }\end{array}$ & $\begin{array}{c}\text { Tiempo } \\
\text { máximo } \\
\text { días }\end{array}$ \\
\hline Pseudomonas & 166 & 5.4 & 2.87 & $5.0-5.8$ & 2 & 20 \\
\hline Xanthomonas & 27 & 6.4 & 3.63 & $5.2-7.6$ & 3 & 13 \\
\hline $\begin{array}{l}\text { Pseudomonas } \\
\text {-Erwinia }\end{array}$ & 125 & 7.4 & 4.17 & $6.7-8.1$ & 3 & 21 \\
\hline Agrobacterium & 4 & & & & 7 & 21 \\
\hline
\end{tabular}

El tiempo mínimo de contaminación, en los segmentos nodales medio y basal de guadua, por los géneros Pseudomonas, Xanthomonas y la asociación ErwiniaPseudomonas (tabla 5; tabla 6), fue de 2 días, siendo más frecuente entre los 2 y 7 días (Figura 1; Figura 2). 


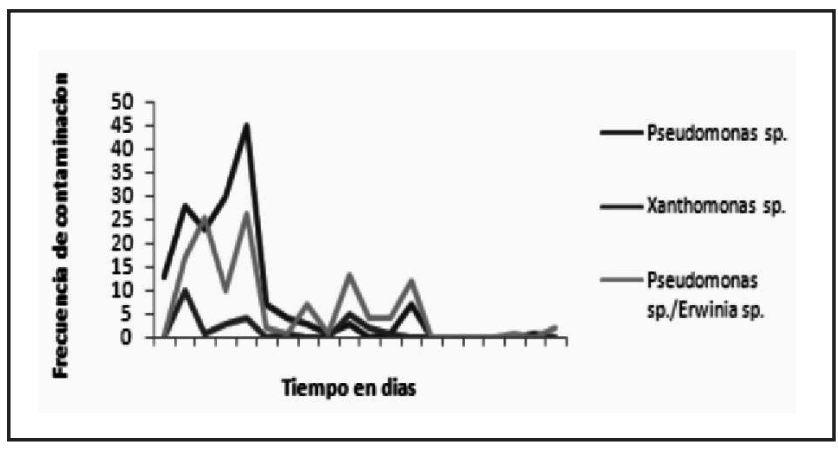

Figura 1. Variación en tiempo de contaminación por bacterias en segmento nodal medio in vitro de $\mathrm{G}$. angustifolia.

\section{PRUEBAS DE SUSCEPTIBILIDAD}

Los cuatro géneros bacterianos identificados (tabla 7), presentaron sensibilidad a los antibióticos: Vancomicina, Amikacina y Gentamicina; en la realización de estas pruebas, Agrobacterium mostró sensibilidad a Cefotaxima; mientras que Xanthomonas, Pseudomonas y Erwinia, exhibieron

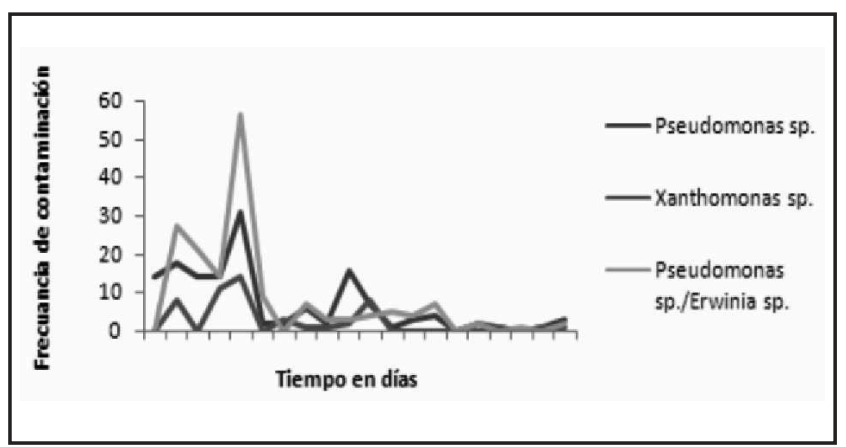

Figura 2. Variación en tiempo de contaminación por bacterias en segmento nodal basal in vitro, de $G$. angustifolia

sensibilidad intermedia, por lo que aquellos antibióticos (Vancomicina, Amikacina y Gentamicina), son los más adecuados para su utilización en el control de dichas bacterias. Agrobacterium, Erwinia, y Pseudomonas, presentaron resistencia a la presencia de Eritromicina; mientras que Xanthomonas presentó sensibilidad a dicho antibiótico.

Tabla 7. Antibiograma: prueba que determina la sensibilidad de las bacterias a diferentes antibióticos.

\begin{tabular}{|l|c|c|c|c|c|c|c|c|c|c|c|c|c|c|}
\hline \multicolumn{1}{|c|}{ GENERO } & C TX & EM & CIP & P & VA & CF & AMC & GE & NO R & AK & AXA & A & SAM & C TR \\
\hline Xanthomonas sp. & SI & S & R & R & S & S & S & S & R & S & R & S & S & S \\
\hline Agrobacterium sp. & S & R & SI & S & S & S & S & S & R & S & R & S & S & R \\
\hline Erwinia sp. & SI & R & R & R & S & R & R & S & S & S & R & R & R & S \\
\hline Pseudomonas sp. & SI & R & S & R & S & R & R & S & S & S & R & R & R & R \\
\hline
\end{tabular}

SI: Sensibilidad intermedia; S: Sensibilidad; R: Resistencia

$\begin{array}{ll}\text { CTR: Ceftriaxona }(30 \mathrm{mcg}) & \text { EM: Eritromicina (15 mcg) } \\ \text { P: Penicilina (10 mcg) } & \text { VA: Vancomicina (30 mcg) } \\ \text { AMC: Amoxicilina } & \text { Ac. clavulonico }(20 / 10 \mathrm{mcg} \text { ) } \\ \text { NOR: Norfloxacino }(10 \mathrm{mcg}) & \text { AK: Amikacina }(30 \mathrm{mcg}) \\ \text { A: Ampicilina }(10 \mathrm{mcg}) & \text { CTX: Cefotaxima }(30 \mathrm{mcg})\end{array}$

CIP: Ciprofloxacino (5 mcg)

CF: Cefalotina (30 $\mathrm{mcg}$ )

GE: Gentamicina (10 mcg)

AXA: Amoxicilina (25 mcg)

SAM:Ampicilina-sulbactam (10/10 mcg)

\section{CONCLUSIONES}

Los géneros de bacterias: Xanthomonas, Erwinia, Pseudomonas y Agrobacterium, son los causantes de la contaminación, en la fase de establecimiento in vitro, de segmentos nodales de Guadua angustifolia.

Pseudomonas y la asociación Pseudomonas-Erwinia, fueron las mayores contaminantes en segmentos nodales medio y basal de guadua in vitro.
Agrobacterium, no indicó niveles de contaminación importantes, que obliguen medidas de control.

\section{RECOMENDACIONES}

Los antibióticos: Vancomicina, Amikacina y Gentamicina, se convierten en herramienta para la prevención y el control del complejo bacteriano, como es el caso de manejo de plantas madre de Guadua angustifolia. 


\section{AGRADECIMIENTOS}

Los autores expresan sus agradecimientos al Laboratorio de Biotecnología del Centro Nacional para el Estudio del BambúGuadua, de La Corporación Autónoma Regional del Quindío; al Centro de Investigaciones Biomédicas "Manuel Elkín Patarroyo" de la Universidad del Quindío; y al Centro de Estudios e Investigaciones en Biodiversidad de la Universidad del Quindío, CIBUQ.

\section{BIBLIOGRAFÍA}

(1) Roca W, Mroginski L. Cultivo de tejidos en la agricultura. Fundamentos y aplicaciones. Centro Internacional de agricultura tropical. CIAT. Cali. Colombia. 1993;19-40 p.

(2) Kyte L, Kleyn J. Plants From Test Tubes. An Introduction to Micropropagation. Third edition. Timber Press. Oregon. USA. 1999.

(3) Digonzelli P, Díaz L, Carrizo S, Lafite J, Sosa S. Diferentes dosis de PPM para controlar la contaminación bacteriana y sus efectos sobre el crecimiento in vitro de la caña de azúcar en la etapa de multiplicación. Facultad de Agronomía y Zootecnia Universidad de Tucumán. Argentina. 2001.

(4) Madigan M, Martinico J, Parker J. Brock. Biología de los microorganismos. 10e edición. Editorial Pearson. España. 2004;369 $-380 \mathrm{p}$.

(5) Leifert C, Ritchie J, Waites W. Contaminants of plant tissue and cell cultures. World Journal of Microbiology and Biotechnology. Oxford. Estados Unidos. 1991;452-469 p.

(6) Giraldo E, Sabogal A. Propagación de la Guadua angustifolia Kunth, método de chusquín. Centro Nacional para el Estudio del Bambú -Guadua. Nota Técnica N 4. C.R.Q. Armenia, Quindío. 1999.

(7) Marulanda ML, Gutiérrez L. Desarrollo de métodos de propagación in vitro y conservación de germoplasma de Guadua angustifolia Kunth. Universidad Tecnológica de Pereira, Colombia. 2003; 7 p.

(8) Manzur D. Bosques de guadua (Bambusa) en laboratorio. En Guaduas y Bosques de Colombia. Revista Agricultura Tropical. 1989.

(9) Marulanda ML, Gutiérrez LG, Márquez MP. Micropropagación de Guadua angustifolia Kunth. Actualidad Biológica. 2005;27(82):5-15.

(10)Jiménez V, Castillo J, Tavares E, Guevara E, Montiel M. In vitro propagation of the neotropical giant bamboo, Guadua angustifolia Kunth, through axillary shoot proliferation. Plant Cell, Tissue and Organ Culture. 2006;86:389-395.

(11)Marulanda ML, Gutiérrez LG, López R. Multiplicación de brotes de Guadua angustifolia Kunth, mediante el sistema de inmersión temporal. (SIT). Grupo de Biodiversidad y Biotecnología. Facultad de Ciencias Ambientales. Universidad Tecnológica de Pereira. Simposio Internacional de Guadua. 2004;7 p.

(12)Jiménez V, Castillo J, Tavares E, Guevara E, Montiel M. Micropropagación de Guadua angustifolia Kunth. a partir de explantes nodales. Simposio Internacional de Guadua. Pereira. Colombia. 2004;10 p.

(13)López R. Informe de actividades en el Laboratorio de Biotecnología del Centro Nacional para el Estudio del Bambú-Guadua. Corporación Regional del Quindío. Colombia. 2006.15p.

(14)López R. Micropropagación de Guadua angustifolia Kunth en medio sólido y por Inmersión Temporal, y estudio de su desarrollo en campo. Trabajo de Grado Magíster. Universidad del Quindío, Universidad Tecnológica, Universidad de Caldas. Armenia, Quindío, Colombia. 2007.

(15)Cruz M, García Y, Sánchez C, Alvarado Y, Acosta M, Roque B, Leiva, M Freire M. Identificación y control de Bacillus sp., contaminante del establecimiento in vitro de Guadua angustifolia Kunth. Biotecnología Vegetal. 2007; Vol 7, № 1:9-13

(16)Murashige T, Skoog F. A revised medium for rapid growth and bioassays with tobacco tissue cultures. Physiol. Plant. 1962 ;15:473-497.

(17)MERCK. Manual de medios de cultivo. Laboratorio Químico-Farmacéutico Merck. Darmstadt, Alemania. 1994.

(18)Koneman EW, Stephen DA, Williams MJ, Schrenberger PC, Washington CW. Diagnóstico Microbiológico. Buenos Aires, Argentina: Editorial Médica Panamericana. 1999;p. 388-461.

(19)Castaño J. Principios básicos de fitopatología. 2a Edición. Centro de Recursos Didácticos (CERED). Departamento de Protección Vegetal. Escuela Agrícola Panamericana. Honduras. 1994;70-84 p. 
(20)Kado C, Heskett M. Selective media for isolation of Agrobacterium, Corinebacterium, Erwinia, Pseudomonas and Xanthomonas. Department of Plant Phatology, University of California. Davis, California. 1970;969-976 p.

(21)Koneman E, Allen S, Dowell VR, Sommers H. Diagnóstico microbiológico. Editorial Médica Panamericana. Buenos Aires Argentina. 1983;11-230 p.

(22)López RM, Gilchrist L, Leyva G. Evaluación de medios de cultivo para el incremento de inoculo de Stagonospora nodorum. Memorias del XXIX Congreso Internacional de la Sociedad Mexicana de Fitopatología, A.C. Monterrey, Nuevo Leon, Mexico. 2002. Abstract F-94.

(23) Rodríguez J, Canton R, Sánchez J, Gómez L, Martínez L, Rodríguez C, Vila J. Procedimientos en microbiología clínica. Recomendaciones de la sociedad española de enfermedades infecciosas y microbiología Clínica. Métodos básicos para el estudio de la sensibilidad a los antimicrobianos. 2000;26p.

(24) Koneman EW. Diagnostico Microbiologico Texto y Atlas a color. 6 edicion. Medica Panamericana. 2006.

(25)Sánchez M, Marmolejo F, Bravo N. Microbiología y aspectos fundamentales. Universidad Nacional. Sede Palmira, Colombia. 2002.142-159p.

(26) Ávila C. Manual de laboratorio de fitopatología. Universidad Pedagógica y Tecnológica de Colombia. 2004; 134p.

(27)Stuart W. Microbiología. Editorial McGraw-Hill Interamericana. México, D.F. 2001;134-147 p.

(28)Walter W, Macbee R. Microbiología general. Editorial Continental S.A. México, D.F. 1972;111-413 p.

(29)Agrios G. Fitopatología. (2a Edición) UTEHA. Noriega Editores. México, D.F. México. 1995.

(30)Surga R, Guevara J. Desinfection trials o control bacterial contamination in in vitro culture of banana (Musa AAA) apexes. Fitopatol. Venezuela. 1994;5 p. 